\title{
Healthcare Policy Four Years On
}

His issue of Healthcare Policy/Politiques de Santé is my last as
editor-in-chief. Launching the journal four years ago was a Field of Dreams
experience - although less a case of "If we build it, they will come" than "If we build it, will they come?" Morris Barer, then Scientific Director of the CIHR Institute of Health Services and Policy Research, was the chief dreamer. The mission was to establish a Canadian journal of health services, policy and management research that responded to the needs of both researchers and decision-makers. My job was to lead the construction crew.

We needn't have worried. They came. Several thousand e-mails, 301 submitted manuscripts, 15 regular issues, one special issue, 142 published peer-reviewed papers, numerous editorials, columns, interviews, commissioned papers, case studies and one lone book review later, Healthcare Policy is a reality. The journal has been accepted for listing in PubMed Central. We've published submissions from across and outside Canada, in French and English, and on topics ranging from First Nations health, primary healthcare, cancer care and health promotion to healthcare financing, pharmaceutical policy, equity of healthcare use and health human resources planning. Research methods have been as varied as the topics and have included operations research, simulation experiments, policy analyses, case studies, surveys, focus groups, health technology assessments, international comparative studies and analyses of administrative and population survey data sets. During the first four years, the journal's manuscript acceptance rate has hovered around 50\%.

Healthcare Policy now has 698 individual and 180 institutional subscribers. Most of the latter are universities and colleges (45\%), government organizations (19\%), healthcare organizations $(16 \%)$ and research organizations $(9 \%)$, through which a large but unknown number of individuals gain access to the journal. Two-thirds of the individual subscribers receive their subscription as a benefit of membership in the Canadian Association for Health Services and Policy Research (CAHSPR). Nonsubscribers can download copies of individual papers from the publisher's website. Some content is available free of charge from the date of publication; downloads of the remaining papers can be purchased. All content becomes open access one year after publication. As of January 8, 2009, downloads of papers published in Healthcare Policy totalled 48,762, typically running between 2,000 and 4,000 per issue - more for open-access papers that have been available longer. In the absence of appropriate comparators, it's hard to know whether to be heartened or dismayed by these data. As an inveterate optimist, I'm inclined to see the glass as half full, but the ambiguity of the numbers points to the need for identifying benchmarks and targets. 
Healthcare Policy seeks to "bridge the worlds of research and decision-making by presenting research, analysis and information that speak to both audiences." Accordingly, we've emphasized applied research and knowledge translation and included such features as selected Promising Practices and Evidence Boosts from the Canadian Health Services Research Foundation and Health Technology Briefs, which primarily target decision-makers. Our review and editorial processes include both researchers and decision-makers. The extent to which we have succeeded in "speaking to both audiences" is hard to gauge. Spontaneous feedback, although almost invariably positive, has been remarkably sparse. Respondents to the publisher's two online surveys have offered helpful suggestions and solid endorsement of what we're doing, but the low response rate doesn't warrant firm conclusions. Clearly, we will need to be more imaginative and systematic in soliciting the perceptions of our readers (and potential readers) if we want those perceptions to inform our editorial decisions.

What else needs work? Our manuscript review process remains creaky. For example, the median time from manuscript submission to reviewer selection is more than 30 days, and from receipt of completed reviews to author notification of a decision is about 40 days. The median time for completion of a full set of reviews is two months. Although we try to obtain at least two academic reviewers and one decision-maker reviewer for each manuscript, we are not always successful and sometimes settle for two reviews. Delays and bottlenecks are inevitable in a process that relies so heavily on unpaid volunteers as both editors and reviewers, but improvement is needed and should be possible.

Healthcare Policy reflects the collective efforts of its authors, reviewers, editors and production staff. Wéve been blessed with dedication and competence in all categories. I particularly want to express my gratitude to Senior Editors François Béland and Rick Roger, Editors Raisa Deber, John Horne, Joel Lexchin, Claude Sicotte, Robyn Tamblyn and Christel Woodward, Editorial Advisory Board Chair and regular columnist Bob Evans and Managing Editors Ania Bogacka and Rebecca Hart for their commitment, good humour and forbearance.

Healthcare Policy is launched and underway but, as English sailor and explorer Francis Drake observed over four centuries ago: "There must be a beginning of any great matter, but the continuing unto the end until it be thoroughly finished yields the true glory." (Dispatch to Francis Walsingham, May 17, 1587, in Navy Records Society, vol. II, 1898)

The voyage may never be "thoroughly finished," but I'm certain the best is yet to come.

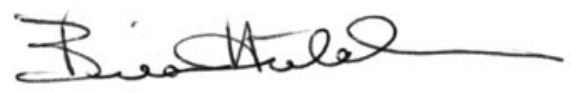

BRIAN HUTCHISON, MD, MSC, FCFP

Editor-in-chief 


\section{Politiques de santé : quatre ans déjà}

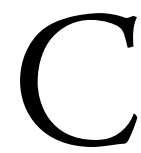

\e tirage de Politiques de Santé/Healthcare Policy est mon dernier numéro à titre de rédacteur en chef. Le lancement de la revue, il y a quatre ans, était une expérience digne de Jusqu'au bout du rêve - bien que dans ce cas, la célèbre réplique « si vous le construisez, ils viendront » serait plutôt « si vous le construisez, viendront-ils? » Morris Barer, alors directeur scientifique de l'Institut des services et des politiques de la santé des IRSC, en était le principal rêveur. L'idée était de créer une revue canadienne consacrée à la gestion, aux politiques et aux services de santé, qui réponde aux besoins des chercheurs et des décideurs. Mon travail consistait à diriger l'équipe pour sa mise en place.

Nous n'aurions pas dû nous préoccuper. Ils sont venus. Politiques de Santé est bel et bien une réalité : plusieurs milliers de courriels reçus, 301 manuscrits présentés, 15 numéros réguliers, un numéro spécial, 142 articles revus par les pairs et publiés, de nombreux éditoriaux, entretiens, articles commandés, études de cas et une critique de livre. La revue a été admise pour la base de données PubMed Central. Nous avons publié des textes du Canada et de l'étranger, en français et en anglais, sur de nombreux sujets dont la santé des communautés autochtones, les services de première ligne, les soins contre le cancer, la promotion de la santé, le financement des services de santé, les politiques sur les produits pharmaceutiques, l'équité des services de santé et la planification des ressources humaines en santé. Les méthodes de recherche employées ont été aussi variées que les sujets traités et ont inclu : recherches opérationnelles, expériences de simulation, analyses de politiques, études de cas, sondages, groupes de discussion, évaluations des technologies de la santé, études comparatives internationales et analyses de données administratives et de sondages auprès des populations. Au cours des quatre premières années, le taux d'acceptation des manuscrits a été d'environ $50 \%$.

Politiques de Santé compte maintenant parmi ses abonnés 698 personnes et 180 institutions. La plupart de ces dernières sont des universités (45\%) et des organismes gouvernementaux (19\%), de santé $(16 \%)$ ou de recherche $(9 \%)$, où un grand nombre de lecteurs peuvent consulter la revue. Deux tiers des personnes abonnées reçoivent la revue comme avantage en tant que membre de l'Association canadienne pour la recherche sur les services et les politiques de la santé (ACRSPS). Les personnes qui ne sont pas abonnées peuvent télécharger des articles à partir du site de la maison d'édition. Certains textes sont gratuits dès leur publication, les autres articles peuvent être achetés. Tous deviennent universellement accessibles un an après la publication. En date du 8 janvier 2009, 48762 téléchargements d'articles de Politiques de Santé 
ont été effectués, soit entre 2000 et 4000 par numéro, et plus pour les articles qui sont accessibles depuis plus longtemps. En l'absence de données de comparaison, il est difficile de savoir si ces chiffres sont réjouissants ou déconcertants. Inlassable optimiste, je suis plutôt porté à voir le verre à moitié plein, mais l'incertitude face aux chiffres fait ressortir la nécessité de définir des repères et des cibles.

Politiques de Santé a comme objectif d'établir des liens entre le monde de la recherche et celui de la décision en présentant des recherche, des analyses et de l'information qui sadressent aux deux milieux à la fois. En ce sens, nous avons mis l'accent sur la recherche appliquée et sur le transfert de connaissances et nous avons inclus des publications telles que Pratiques prometteuses et Données à l'appui, de la Fondation canadienne de la recherche sur les services de santé, et Coup d'cil sur les technologies de la santé, qui visent principalement les décideurs. Notre système de révision et d'édition fait appel à des chercheurs et à des décideurs. Il est cependant difficile dévaluer à quel point nous sommes parvenus à toucher les deux auditoires visés. Les commentaires spontanés, bien que presque toujours favorables, ont été extrêmement rares. Les réponses aux deux sondages en ligne de la maison dédition ont été très utiles et encourageants, mais le faible taux de réponse ne permet pas de tirer des conclusions définitives. Si nous voulons que notre ligne éditoriale tienne compte de l'opinion des lecteurs (actuels et potentiels), nous devrons certainement être plus systématiques et redoubler d'imagination afin d'obtenir cette information.

D'autres aspects restent à peaufiner. Le processus d'évaluation des manuscrits est encore fragile. Par exemple, le temps médian entre la proposition d'un manuscrit et le choix de ses réviseurs est de plus de 30 jours, et celui entre la réception des révisions et l'annonce de la décision à l'auteur est d'environ 40 jours. Le temps médian pour la révision d'un ensemble d'articles est de deux mois. Bien que nous tentions d'avoir au moins deux universitaires et un décideur pour chaque manuscrit, nous ne sommes pas toujours en mesure de le faire. Quand on compte sur le travail bénévole des éditeurs et des réviseurs, les délais sont inévitables, mais il y a certainement place à amélioration.

Politiques de Santé est le fruit de l'effort collectif des auteurs, des réviseurs, des éditeurs et du personnel. Nous avons été choyés par tous en termes de dévouement et de savoir-faire. Je tiens personnellement à remercier les éditeurs principaux, François Béland et Rick Roger, les éditeurs Raisa Deber, John Horne, Joel Lexchin, Claude Sicotte, Robyn Tamblyn et Christel Woodward, le président du comité de rédaction et éditorialiste Bob Evans et les directrices de rédaction Ania Bogacka et Rebecca Hart pour leur dévouement, leur bonne humeur et leur patience.

Politiques de Santé est bien sur sa lancée, mais comme l'observait, il y a quatre siècles, le navigateur et explorateur anglais Francis Drake : «Il y a un commencement à tout grand accomplissement, mais ćest le chemin qui conduit à son achèvement qui 
mène à la véritable gloire. » (Adressé à Francis Walsingham, 17 mai 1587, dans Navy Records Society, vol. II, 1898.)

Le voyage ne sera sans doute jamais achevé, mais je suis convaincu que le meilleur reste à venir.

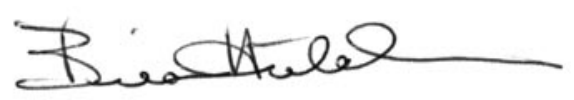

BRIAN HUTCHISON, MD, MSC, FCFP

Rédacteur en chef

\section{HEALTH INNOVATION AND POLICY SUMMIT}

\section{Driving Healthcare Forward in Leaner Times}

April 27 - 28, 2009 | St. Andrew's Club and Conference Centre | 150 King Street West, Toronto 2009 Program Highlights:

- A Renewed Approach to Healthcare Funding - Emerging Strategies

- Setting the Policy and Regulatory Agenda for New Technologies

- Implementation Plan and Timetable for Aging at Home Strategy

- Assessing the Impact of Privately Owned Clinics on Health Services Delivery

- Pharma Spending - How to Rein in Costs While Meeting Growing Needs?

- Healthcare P3s - Lessons Learned and the Way Ahead and much more...
Dr. Dana Hanson

President Elect

World Medical Association

Scott Dudgeon

CEO

Alzheimer's Society

of Canada

Bronze Sponsors

国 Best Doctors

Microsoft

Marketing Partners

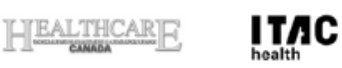

ITAC

ENROLL TODAY! Call 1888 777-1707 or fax 1866 777-1292

Register online at www.insightinfo.com

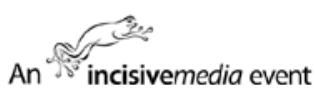

